\title{
Nutrition and Dementia
}

\author{
Y. Brockdorf ${ }^{\prime}$, J.E. Morley ${ }^{2}$ \\ 1. Dietitian, Nutrition for Success, LLC, Hillsboro, Oregon, USA; 2. Division of Geriatric Medicine, St Louis University School of Medicine, St Louis, MO USA
}

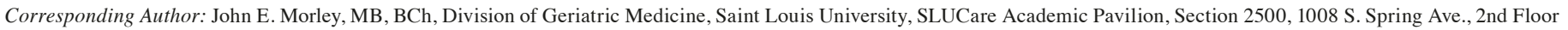
St. Louis, MO 63110,Email: john.morley@health.slu.edu,Twitter: @drjohnmorley

Key words: Nutrition, dementia, diet, antioxidants, Mediterranean diet.

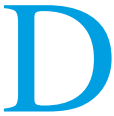
ementia is a chronic, often age related and irreversible condition which leads to cognitive decline, inability to memorize new information and recall previously stored memories. The World Health organization reports that every year 7.7 million new cases of dementia are diagnosed and project that by 2050 there will be over 100 million people living with dementia (1). There are several theories and multiple causes of dementia. In the 1900 s, atherosclerosis was considered to be the main cause of cognitive decline and dementia. Almost a century after, $\mathrm{A} \beta$ peptide was discovered along with $\mathrm{B}$ amyloid plaques. A year after, in 1981 another theory was established and it involves cholinergic deficiency in patients with Alzheimer disease. It was noted that acetyl cholinergic neurons in the forebrain are degenerating, however, subsequent research has shown that other neurotransmitters are also responsible. So far, the established causes for dementia are: Primary age-related tauopathy, hippocampal sclerosis, vascular dementia, Lewy body dementia and Alzheimer disease (2).

Nutrition plays a very large role in the onset and prognosis of dementia. It is one of the key lifestyle changes in the strategies to combat dementia (3). The brain is an organ which requires a disproportionate amount of energy compared to its mass. In normal individuals this energy comes solely from glucose. Glucose provides all the energy needed for normal function of the brain. This includes cellular maintenance as well as neurotransmitter production (4). Dietary lipids are a key component of neural membranes. Special fats such as gangliosides and sphingolipids are responsible for normal neural impulse conductivity and as such important for cognition. Several studies show, that amino acid intake can be a significant factor in dementia incidence and prognosis. In a study which monitored choline intake in 2497 subjects over 22 years, it was concluded that patients with higher choline intake had a lower incidence of dementia and cognitive impairment was milder (5). In another study 4 amino acids (leucine, phenylalanine, lysine and threonine) played a role in the maintenance of cognitive function over 8 years (6).

There are many studies exploring the connection between different fatty acids and dementia. A review study exploring the relationship between saturated and trans-fats and dementia found that people who ingested more saturated fatty acids and trans-fats showed greater cognitive decline than controls. The exact mechanism was not discerned; however, the authors suspected that saturated fatty acids and trans fats increased the LDL level, leading to accelerated atherosclerosis and so promoted the onset of vascular dementia (7). Fatty acids impact cognition in several ways. Consumption of saturated fatty acids can accelerate atherosclerosis, promote thrombogenesis and impair fibrinolysis (8). This promotes the events of vascular dementia as reduced blood flow and microinfarctions can cause long lasting hypoxia of the brain $(1,2,9)$. Saturated fatty acids also increase the insulin resistance, which leads to type II diabetes mellitus (8). Diabetes mellitus itself is a risk factor for dementia (3).

After feeding, subjects found their ability to memorize improved (10). Cholecystokinin activates the ascending fibers into the hypothalamus and improves ability to memorize. Ghrelin, a hormone which is elevated in starvation, improves ability to remember. However prolonged starvation has an adverse effect on cognition, due to decrease of leptin levels in the blood. Leptin is another hormone produced by adipocytes when well sated.

\section{Micronutrients}

Micronutrients are substances essential to life which cannot be synthesized by the human body. They are often required in minute amounts and are acquired either through diet, or through vitamin supplements. Micronutrients usually serve as coenzymes or are a key part in some enzyme structures. The most common micronutrients which are included in enzyme structures are copper, selenium, manganese and zinc. Antioxidant vitamins are vitamin $\mathrm{C}$, carotenoids, flavonoids and most important vitamin E. They are an important component of antioxidant system, and they fight oxidative stress. A number of studies have linked different micronutrients to prognosis of dementia and aging (11).

Blueberries are an important source of flavonoids (polyphenols), which are potent dietary antioxidants. After daily blueberry supplementation for 16 weeks, participants showed increased activity in left pre-central gyrus, left middle frontal gyrus and left interior parietal lobe (12). Daily supplementation of wild blueberries has boosted the executive function, short- 
term memory, mood and reaction time in children aged 7-10 (13). In adults, supplementation of blueberries showed improved paired associate learning, word list recalling and reduction of depressive symptoms (14).

Persons with low serum vitamin D had lower cognition than controls. The subjects were persons with significant vitamin D deficiency $(<25 \mathrm{nmol} / \mathrm{L})$, while controls had sufficient levels of vitamin D $((\geq 75 \mathrm{nmol} / \mathrm{L})$. The authors calculated that adjusted relative risk for decline on Mini-Mental State Examination (MMSE) was 1.60 (95\% CI, 1.19-2.00) in patients with low vitamin D levels. They estimated a drop of 0.3 points on MMSE scale for every year people had vitamin D deficiency (15). It is believed that vitamin $\mathrm{D}$ reduces production of $\beta$-amyloid in the brain. An adverse event in vitamin D supplementation may be consequent hypercalcemia. When given higher vitamin D supplementation doses, no cognitive improvement was detected. It is believed that excess calcium due to vitamin D over-supplementation has adverse effects on cognition more than vitamin $\mathrm{D}$ benefits it (16).

\section{Diet}

Diet has long been considered one of the main aspects of health. Diet influences many diseases including cardiovascular disease, diabetes and cancer. Recent studies seem to suggest that diet has a role in neurodegeneration as well. Western diet is defined as a regular consumption of roast meat, sausages, hamburgers, steak, chips, crisps and sugary drinks, over fresh vegetables, fruit and fish. It was reported that people on western diets had an average of 45.7-52.6 $\mathrm{mm}^{3}$ smaller hippocampus volume, compared to people on a healthy diet (17).

This healthy diet involving fruits, vegetables, and fish, with lowered intake of dairy, red meat, and sugars can be called a Mediterranean diet. This diet was based on leafy greens, fruits and vegetables, whole grains, legumes, seeds and nuts, and it was linked to several benefits of those populations, such as higher than average life expectancy, lower rates of cancer and some cardiovascular diseases. Mediterranean diet's most distinctive feature is that it features minimal red meat and dairy intake, with most of the fat coming from olive oil. The phenyls in olive oil enhance cognition and decrease oxidative damage in the brain (18). In general, these foods are rich with vitamins such as folates and B vitamins, without substances such as refined sugars, cholesterol and trans fats. There is evidence that suggests that Mediterranean diet could be beneficial for many conditions, including those of mental decline. People on Mediterranean diet tend to have less chronic illnesses, lower rates of cognitive decline, lower incidence of Alzheimer's disease and better cognition $(19,20)$. A study investigating effects of Mediterranean diet on grey and white matter volumes found no change in grey matter volumes. However, they reported a gain in structural connectivity with strong cognitive benefits in people on Mediterranean diet (21).

In addition to the Mediterranean diet, Dietary Approach to Systolic Hypertension (DASH) diets have been shown to slow cognitive decline as well. An experimental, MIND diet is a diet which combines these two diets together, and yields promising results. The MIND diet requires additional intake of berries and green leafy vegetables, but does not specify intake of fruit. Similar to Mediterranean diet, it is reported to slow cognitive decline with age (22).

\section{Curcumin}

One novel substance that is showing promising results in treatment and prevention of dementia is curcumin. Since curcumin is lipophilic, it passes through cell membranes where it expresses a variety of effects. By entering microglia cells, curcumin reduces neuroglial and microglial proliferation and differentiation. Because chronic activation of microglia exacerbates $\beta$ amyloid plaques, by reducing microglial proliferation, curcumin reduces the $\beta$ amyloid plaque generation. Curcumin reduces astrocyte proliferation as well, but increases differentiation of oligodendrocytes and as such improves myelogenesis $(23,24)$. Curcumin inhibits amyloid $\beta$ aggregation through downregulating BACE1 expression. BACE1 is an enzyme which cleaves Amyloid Precursor Protein to AB. Another mechanism of AB is through GSK-3B presenilin 1 activation. Administration of curcumin reduced $A B$ production by reducing levels of Presenilin-1 and GSK$3 \mathrm{~B}$. Besides lowering the production of $\mathrm{AB}$, studies show that curcumin inhibits deposition of amyloid $\beta$ in cell membranes. Studies also show that curcumin inhibits hyperphosphorylation of the tau protein and lowers the copper deposition in amyloid plaques in people with Alzheimer disease (24). Curcumin also shows immediate cognitive effects. Acute treatment with curcumin improved cognition in three subtraction tasks by $16 \%$ (25).

Despite its advantages, curcumin has naturally low bioavailability. When taken orally, it shows poor absorption in the plasma, either through low intestinal absorption or rapid metabolism. In order to be properly absorbed, curcumin has to be bound to a carrier, or it could be administered as a curcumin $\operatorname{analog}(24)$.

\section{Cinnamon}

Cinnamon as a condiment has been so far associated with various health benefits such as antioxidant, antimicrobial, antiviral and insulin like activities. Cinnamon has also shown an ability to interact with tau aggregation in vitro, reducing the tau protein content and increasing the tau soluble fraction. This effect suggests that cinnamon may inhibit the formation of tau fibers. In addition, even in already formed fibers, treatment with cinnamon can reverse the fiber formation and induce fiber disassembly $(26,27)$.

However, similar to curcumin, cinnamon active ingredients, namely cinnamaldehyde have poor bioavailability $<20 \%$ and low water solubility (27).

\section{Discussion}

Mediterranean diet focuses more on essential nutrients, which is why it seems better in comparison to Western diet 
$(17,19,20)$. Fruits in this diet are a rich source of fructose (sweeter than sucrose but less calories for the same sweetness) and contains a lot of fiber, polyphenyls and anthocyanins which are potent antioxidants $(12,28)$. In addition, fruits are hosts to a variety of vitamins, most notably vitamin $\mathrm{C}$, folic acid and dietary fiber $(28,29)$. By reducing cholesterol absorption, dietary fibers delay atherosclerosis, thereby delaying vascular aspects of dementia [8]. Folic acid is essential for homocysteine metabolism and is directly linked to lower risk of dementia (3, 5). Vitamin $C$ has also been linked to reduced dementia risk $(3,11)$. Vegetables in Mediterranean diet are also a rich source of dietary fiber and carotenoids, which have also been found to reduce the risk of dementia $(19,20,30)$. Vegetable oil and seeds are a rich source of mono and poly unsaturated fatty acids (31). Soybean, flaxseed, black currant and rapeseed are rich with omega 3 fatty acids, and so are their oils (32). The most common monounsaturated fatty acid, oleic acid, can be found in high quantities in olive oil, which is a characteristic for Mediterranean diet. Fish meat and other seafood are also very rich in omega 3 fatty acids and vitamin $\mathrm{E}$, which reduce oxidative stress in the brain $(11,31)$. On the other hand, diets rich in red meat, poultry and dairy have an excess amount of saturated fatty acids, mainly are myristic, palmitic and stearic acid which are linked to higher cholesterol synthesis in the liver $(8,17,31)$. By raising LDL cholesterol, saturated fatty acids promote atherosclerosis and in turn vascular aspects of dementia $(1,8)$. Saturated fatty acids also increase resistance to insulin leading to type II diabetes (8). A high sugar diet also leads to increased insulin resistance and causes diabetes $(3,4)$. Then, unregulated blood sugar causes endothelial dysfunction which promotes atherosclerosis (33). In addition to atherogenic effect, high blood glucose also causes glucotoxicity, and therefore further reducing blood flow to the brain $(4,33)$. An excess of sugar is converted into saturated fatty acids which in turn leads to production of LDL cholesterol and provides another path, besides glucotoxicity to atherosclerosis $(8,31$, $33)$.

Cognitive decline is much greater in those who eat mostly high sugar, high saturated fat/trans-fat diets with not enough fiber and vitamins in the diet. Mediterranean diets, rich in fruit, vegetable, fiber, polyphenyls and omega 3 fatty acids seem to be associated with lower incidence of dementia and lower cognitive decline in dementia and old age. Since the effects of poor diets are cumulative and take a long time, it would be prudent to switch to a Mediterranean diet sooner in life.

Conflict of interests: The authors report there are no conflicts of interest regarding this manuscript.

\section{References}

1. Iadecola C. The Pathobiology of Vascular Dementia. Neuron. 2013;80.

2. Morley JE, Farr SA. Alzheimer Mythology: A Time to Think Out of the Box. AMDA. 2016.

3. Pinnila FG. Brain foods: the effects of nutrients on brain function. Nat Rev Neurosci. 2008 Jul;9(7):568-578.

4. Mergenthaler P, Lindauer U, Dienel GA, Meisel A. Sugar for the brain: the role of glucose in physiological and pathological brain function Trends Neurosci. 2013 Oct;36(10):587-597.

5. Ylilauri MP, Voutilainen S, Lönnroos E, Virtanen HE, Tuomainen T, Salonen JT et al. Associations of dietary choline intake with risk of incident dementia and with cognitive performance: the Kuopio Ischaemic Heart Disease Risk Factor Study. Am J
Clin Nutr. 2019;0:1-8

6. Kinoshita K, Otsuka R, Takada M, Tsukamoto-Yasui M, Nishita Y, Tange C, Tomida M, Shimokata H, Kuzuya M, Imaizumi A, Arai H. The Association between Dietary Amino Acid Intake and Cognitive Decline 8 Years Later in Japanese CommunityDwelling Older Adults. J Nutr Health Aging. 2021;25:165-171.

7. Barnard ND, Bunner AE, Agarwal U. Saturated and trans fats and dementia: a systematic review. Neurobiol. Aging. 2014;35:65-73.

8. Calder PC. Functional Roles of Fatty Acids and Their Effects on Human Health. JPEN J Parenter Enteral Nutr. 2015;20(10):1-15.

9. Bennett S, Grant MM, Aldred S. Oxidative Stress in Vascular Dementia and Alzheimer's Disease: A Common Pathology. J. Alzheimer's Dis. 2009;17:245-257.

10. Morley JE. Nutrition and the Brain. Clin Geriatr Med. 2010;26:89-98.

11. Morris MC. Nutritional determinants of cognitive aging and dementia. Proceedings of the Nutrition Society. 2012;71:1-13.

12. Boespflug EL, Eliassen JC, Dudley JA, Shidler MD, Kalt W. Enhanced Neuronal Activation with Blueberry Supplementation in Mild Cognitive Impairment. Nutr Neurosci. 2018 May;21(4):297-305

13. Barfoot KL, May G, Lamport DJ, Ricketts J. The effects of acute wild blueberry supplementation on the cognition of 7-10-year-old schoolchildren. European Journal of Nutrition. 2019;58:2911-2920.

14. Krikoran R, Shidler MD, Nash TA, Kalt W, Vinqvist-Tymchuk MR, Shukitt-Hale B et al. Blueberry Supplementation Improves Memory in Older Adults. J Agric Food Chem. 2010 Apr 14;58(7:3996-4000.

15. Llewellyn DJ, Lang LA, Langa KM, Terrera GM, Phillips CL et al. Vitamin D and Risk of Cognitive Decline in Elderly Persons. Arch Intern Med. 2010 Jul 12; 170(13):1135-1141.

16. Morley JE. Does vitamin D modulate cognition?. Nat. Rev. Neurol. 2014;10(11):613614

17. Jacka FN, Cherbuin N, Anstey KJ, Sachdev P, Butterworth P. Western diet is associated with a smaller hippocampus: a longitudinal investigation. BMC Med. 2015;13:215.

18. Farr SA, Price TO, Dominguez LJ, Motisi A, Saiano F, Niehoff ML, Morley JE, Banks WA, Ercal N, Barbagallo M. Extra virgin olive oil improves learning and memory in SAMP8 mice. J Alzheimers Dis. 2012;28(1):81-92.

19. Hardman RJ, Kennedy G, Macpherson H, Scholey AB, Pipingas A. Adherence to a Mediterranean-Style Diet and Effects on Cognition in Adults: A Qualitative Evaluation and Systematic Review of Longitudinal and Prospective Trials. Front. Nutr . 2016 Jul;3(22):1-13

20. Vinciguerra F, Graziano M, Hagnas M, Frittitta L, Tumminia A. Influence of the Mediterranean and Ketogenic Diets on Cognitive Status and Decline: A Narrative Review. Nutrients. 2020;12:1-22.

21. Pelletier A, Barul C, Feart C, Helmer C, Bernard C, Periot O et al. Mediterranean diet and preserved brain structural connectivity in older subjects. Alzheimers. Dement 2015;11(9):1-9.

22. Nagai K, Koshiba H, Shibata S, Matsui T, Kozaki K. Correlation between the serum eicosapentanoic acid-to-arachidonic acid ratio and the severity of cerebral white matter hyperintensities in older adults with memory disorder. Geriatr Gerontol Int. 2015;15(1):48-52.

23. Mishra S, Palanivelu K. The effect of curcumin (turmeric) on Alzheimer's disease: An overview. Ann Indian Acad Neurol. 2008;11(1):13-19.

24. Tang M, Taghibiglou. The Mechanisms of Action of Curcumin in Alzheimer's Disease. J. Alzheimer's Dis. 2017;58(4):1003-1016.

25. Cox KHM, Pipingas A, Scholey AB. Investigation of the effects of solid lipid curcumin on cognition and mood in a healthy older population. J. Psychopharmacol. 2015;29(5):642-651.

26. Peterson DW, George RC, Scaramozzino F, LaPointe NE, Anderson RA, Graves DJ et al. Cinnamon Extract Inhibits Tau Aggregation Associated with Alzheimer's Disease In Vitro. J. Alzheimer's Dis . 2009;17:585-597.

27. Momtaz S, Hassani S, Khan F, Ziaee M, Abdollai M. Cinnamon, a promising prospect towards Alzheimer's disease . Pharmacol. Res. 2018;130:241-258.

28. Spencer SJ, Korosi A, Laye S, Shukitt-Halle B, Barrientos RM. Food for thought: how nutrition impacts cognition and emotion. NPJ Sci. Food. 2017;7:1-8.

29. Crujeiras AB, Parra DM, Rodriguez C, Martinez de Morentin BE, Martinez A. A role for fruit content in energy-restricted diets in improving antioxidant status in obese women during weight loss. Nutrition. 2006;22(6):593-599.

30. Yuan C, Chen H, Wang Y, Schneider JA, Willett WC, Morris MC. Dietary carotenoids related to risk of incident Alzheimer dementia (AD) and brain AD neuropathology: a community-based cohort of older adults. Am J Clin Nutr 2020;00:1-9.

31. Petrovic S, Arsic A. Fatty Acids: Fatty Acids. Encyclopedia of Food and Health, Elsevier. 2016:623-631

32. Haag M. Essential fatty acids and the brain. Can J Psychiatry. 2003;48:195-203.

33. González-Reyes RE, Aliev G, Ávila-Rodrigues M, Barreto GE. Alterations in Glucose Metabolism on Cognition: A Possible Link Between Diabetes and Dementia. Current Pharmaceutical Design. 2016;22:812-818. 